\title{
Importance of Auditory Verbal Therapy/Speech Therapy after Cochlear Implantation: A Case Study
}

\author{
Anna Vinu Varghese ${ }^{1}$ and A. Turin Martina ${ }^{2}$ \\ ${ }^{1}$ Research Scholar, ${ }^{2}$ Research Supervisor and Associate Professor, PG and Research Department of Rehabilitation Science \\ Holy Cross College (Autonomous), Tiruchirappalli, Tamil Nadu, India
}

\begin{abstract}
Studies in the past years have shown that cochlear implant is the most effective management for profound hearing loss. Majority of the Cochlear Implant recipients are children. With the surgery done after early identification and with intensive speech therapy/ auditory verbal therapy, the speech and language skills as well as the listening skills can be improved. The present study shows the importance of AVT/Speech Therapy in habilitation children with Cochlear Implants by demonstrating the pre and post therapy results of subjective measures done in a single case, even with a gap in habilitation of 1.5 years. The study brings out notable results which correlate with previous studies published.

Keywords: Intensive Speech Therapy, Auditory Verbal Therapy, Cochlear Implant
\end{abstract}

\section{INTRODUCTION}

Cochlear implant is the most wonderful invention of the 20th century. Since the mid-1980s, the cochlear implant has been used for children who have a profound hearing loss. Since the mid-1980s, cochlear implants have been increasingly used in the management of childhood deafness and implantation has gradually gained wider clinical acceptance. The cochlear implant does not amplify the sound as hearing aid does, but it directly stimulates the auditory nerve via electrical impulses, bypassing the hair cells. Dramatic progress has been noted in the perception of speech by children with cochlear implants, which has surpassed that of children with comparable hearing loss who used hearing aids. With cochlear implant surgery done immediately and with adequate language intervention given, the child can acquire normal or near normal speech and language skills and thus the quality of life can be improved.

\section{REVIEW OF LITERATURE}

The primary justification for early identification of hearing impairment in infants relates to its impact on speech and language acquisition, cognitive achievements and social/emotional development.

According to IDEA (Individuals with Disabilities Education Act, 2008), hearing is critical for the development of speech, language, communication skills, and learning. The earlier that hearing loss occurs in a child's life, the more serious is the effect on the child's development. Similarly, the earlier the hearing loss is identified and intervention begun, the more likely it is that the delays in speech and language development will be diminished. Recent research indicates that children identified with hearing loss who begin services before 6 months old develop language (spoken or signed) on a par with their hearing peers.

Delayed identification and management of hearing impairment may impede the child's ability to adapt to family and community life and may mimic or cause behavioural problems or may enlarge the experienced intellectual disability.

Yoshinaga-Itano et al. (1998) compared the language abilities of earlier- and later-identified hearing impaired children. The results indicate that children whose hearing losses were identified by 6 months of age demonstrated significantly better language scores than children identified after 6 months of age. For children with normal cognitive abilities, this language advantage was found across all test ages, communication modes, degrees of hearing loss, and socioeconomic strata. It also was independent of gender, minority status, and the presence or absence of additional disabilities.

Teresa Ching (2015) in her study stated that, early intervention, either in the form of amplification or cochlear implantation, was associated with higher language scores. Early performance predicted later language development.

\section{PURPOSE}

This study aims to examine a cochlear implant recipient's unique experience of rehabilitation and to state the importance of Auditory Verbal Therapy/Speech Therapy after Cochlear Implantation.

\section{METHOD}

This study was conducted through direct interview with the parents and by observing the child's behavior. Test materials to assess the listening, speech and language skills were administered at the time of initial evaluation, along with the case history and after 6 months of intensive therapy under a licensed Auditory Verbal Therapist. The child attended therapy 4 times a week, each session lasting 1 hour. Appropriate parent counseling was given and home training was also given. 
The test materials used were, Infant-Toddler Meaningful Auditory Integration Scale (IT-MAIS), ReceptiveExpressive Emergent Language Scale(REELS), Integrated Scale of Development, Ling's 6 sound test, Intelligibility Rating Scale. Mean length of utterance was also checked.

\section{RESULTS AND DISCUSSION}

Case History form and test materials were administered and the results are stated below:

\section{A. Case History}

The female child was diagnosed with profound hearing loss at the age of 18 months. Since then the child was using bilateral digital hearing aids and was attending speech therapy. As no progress was seen in auditory skills as well as speech and language skills, the parents opted for Cochlear Implantation. CI surgery was done for the right ear on 3rd December 2015 and the device was activated on December 22. The child started attending Auditory Verbal Therapy in the same centre and demonstrated good progress. After 6 months, they discontinued the habilitation due to personal reasons. The child was given no stimulation at home. She started using gestures more. But they continued mapping sessions and timely audiological evaluations. After 1.5 years (December 2017), the child (4.7years) was again taken for auditory verbal therapy, but in a different centre.
Case history was taken and Speech and Language Assessment was done.

B. Pre-therapy Evaluation - Speech and Language Skills as on December 2017

Comprehension and Expression of lexical categories such as body parts, family members, common objects, fruits and vegetables, animals, birds, colors and action verbs were present. But vocabulary in each category was limited to 5-6 common words. Her expressive vocabulary consisted of less than 100 words. The child speaks in 2 word level unclear sentences. She uses 3 word level occasionally. But her speech consisted of nouns and verbs only. It lacked grammatical markers. Her speech was intelligible to family members only.

When her speech was not understood by others, she use gestures. She asks for repetition if she cannot understand what is being said. She demonstrated poor listening skills. She couldn't maintain her attention to short stories consisting of 4-5 word sentences. She enjoys watching television, but she always mute the volume. She tries to avoid situations that demand her to speak. Occasionally she initiates speech with familiar people.

Based on the assessment Speech and Language Therapy was started and considerable improvement is seen in her skills.

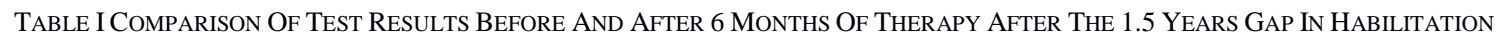

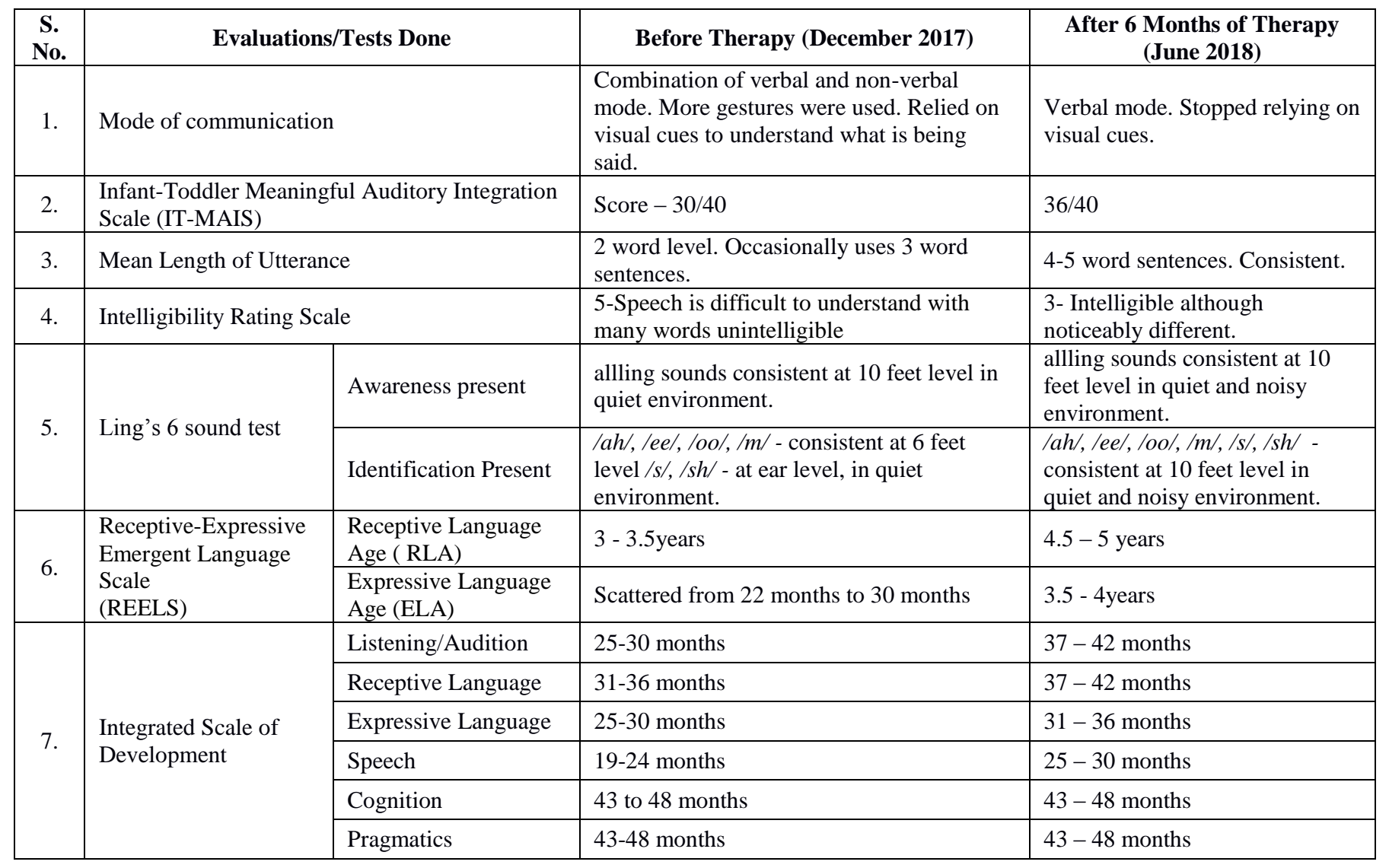




\section{Post-therapy Evaluation-Speech and Language Skills as on June 2018}

Comprehension and Expression of lexical categories such as body parts, family members, common objects, fruits and vegetables, animals, birds, colors and action verbs were present. She comprehends and express gender pronouns, opposites, singular-plurals and pronouns. The vocabulary in each category consisted of 12-15 words. Her expressive vocabulary was increased to $500-1000$ words. The child now speaks in 4-5 word level sentences consistently with grammatical markers. Her speech is now intelligible to people outside the family also. Her listening skill was also improved considerably. She could listen to short stories with 5-6 sentences and retell the story with picture cue cards. She now enjoys watching television with volume. Now she likes meeting new people and initiates conversation with unfamiliar people also.

She is more active and focused than she was before attending regular therapy sessions. Table I show the comparison of test results before and after 6 months of therapy after the 1.5 years gap in habilitation.

The pre and post subjective evaluations show significant improvement in the child's listening and speech and language skills. Significant difference in the performance of the pre and post evaluations reveals the effect of Auditory Verbal Therapy/Speech Therapy even after a gap of 1.5 years in rehabilitation.
Supporting studies indicated that AVT can even help children with HI beyond three years of age to develop age appropriate language skills and catch up with their hearing peers and children receiving AVT can learn to recognize words accurately even in the presence of background noise. (Kaipa and Danser 2015).

\section{CONCLUSION}

Cochlear Implant and Auditory Verbal Therapy has become the gold standard in habilitating children with permanent hearing loss. Though this is a single case study, the results correlate with previous researches with larger sample size. This study reveals that, even after a gap in rehabilitation, the listening, speech and language skills can be improved with intensive therapy.

\section{REFERENCES}

[1] Chowdhry, J. (2010). Auditory Verbal Therapy. Otorhinolaryngology Clinics, An International Journal, 2, 157-160. doi:10.5005/jpjournals-10003-1030

[2] Diller, G., Graser, P. \& Schmalbrock, C. (2001). Early natural auditory-verbal education of children with profound hearing impairments in the Federal Republic of Germany: results of a 4 year study. International Journal of Pediatric Otorhinolaryngology, 60(3), 219-26.

[3] Kaipa, R., \& Danser, M. L. (2016). Efficacy of auditory-verbal therapy in children with hearing impairment: A systematic review from 1993 to 2015. International Journal of Pediatric Otorhinolaryngology, 86, 124-134. doi:10.1016/j.ijporl.2016.04.033. 